\title{
Non-animal approaches in skin toxicology
}

\author{
D. A. Basketter
}

Published online: 19 July 2012

(C) Springer-Verlag 2012

For those of us who are older toxicologists, the great majority of us began our careers at a time when in vitro toxicology seemed to represent something that could only be achieved in a very distant future, almost a piece of science fiction. However, stimulated by ethical and political pressure, in particular in relation to cosmetics (e.g. European Cosmetics Directive/Regulation), there is now a greater urgency to deliver practical solutions in this area of science in a shorter time frame and with the hope, or even expectation, that these achievements will be more than just a fiction. For many of us, it also may have seemed that toxicology endpoints which we viewed as the most simple, such as skin and eye irritation, ought indeed to be capable of being replaced in a relatively short time frame. To some extent, this has occurred, although the quality of the methods in terms of human safety assessment still remains unclear (as was also true of the in vivo methods). Nevertheless, success in this area has meant that focus has now turned to the endpoint of skin sensitisation. Great efforts have been made on this topic in terms of the identification of significant skin sensitisation hazard. For example, work is now underway at the European Centre for the Validation of Alternative Methods European Union Reference Laboratory (ECVAM EURL), which is considering four in vitro alternatives that are already close to the final stages of validation. In addition, it has in hand several other methods for the assessment of their validation status. However, the focus of all of these methods, at least primarily, is to predict the same outcome as the in vivo assays in terms of regulatory hazard classification. Whilst this may be a useful initial step, the protection of human health requires very

D. A. Basketter $(\square)$

DABMEB Consultancy Ltd, Sharnbrook, UK

e-mail: dabmebconsultancyltd@me.com much more, that is, it needs risk assessment and risk management. To achieve these aims, it is necessary not only to identify substances which may be skin sensitisers but also to assess their relative human skin sensitising potency. It is this information that feeds into risk assessment/management. Indeed, it is evident that the current burden of the human skin disease caused by skin sensitising chemicals, allergic contact dermatitis, is caused not by a failure to identify hazards, but rather an insufficient translation of those hazards in terms of risk assessment/ management.

This issue of Archive of Toxicology contains a state of the art review of non-animal approaches to the predictive identification and assessment of potentially skin sensitising chemicals. Bauch and co-workers have presented a very detailed review examining not only the goal of hazard identification, but also the practical possibilities of using current in vitro methods for the purposes of potency prediction. They show that there is now a large body of work focused on the former of these goals, but to date scant attention has been paid to the latter. They are not the first to point out this gap, such that it is clearly timely that this matter receives more attention. Also, addressed is the matter of the combination of methods in order to deliver more accurate predictions, which might apply both to hazard identification and to potency assessment. It is to be hoped that this proceeds with a more balanced focus than was the case for genotoxicity assessment, where the method battery approach tended to deliver sensitivity very much at the expense of specificity (Kirkland et al. 2007).

So, what is the prospect on this occasion for in vitro skin sensitisation alternatives becoming a reality? As Bauch et al. observe, 'No toxicology test is completely perfect....', and this is well accepted within the validation 
process, with the consequence being that, for skin sensitisation, a non-animal hazard identification test within a relatively short time frame now seems entirely possible, at least for a large number of substances. Two key things remain to be seen: whether toxicological science can deliver potency assessments without using animals and whether regulatory toxicologists can accommodate the imperfections of in vitro methods as well as they have seemed able so to do for the in vivo assays.

\section{References}

Kirkland D, Pfuhler S, Tweats D, Aardema M, Corvi R, Darroudi F, Elhajouji A, Glatt H, Hastwell P, Hayashi M, Kasper P, Kirchner S, Lynch A, Marzin D, Maurici D, Meunier JR, Müller L, Nohynek G, Parry J, Parry E, Thybaud V, Tice R, van Benthem J, Vanparys P, White P (2007) How to reduce false positive results when undertaking in vitro genotoxicity testing and thus avoid unnecessary follow-up animal tests: report of an ECVAM workshop. Mutat Res 628:31-55 